\title{
The 80-W KTP GreenLight laser vaporization of the prostate versus transurethral resection of the prostate (TURP): adjusted analysis of 5-year results of a prospective non-randomized bi-center study
}

\author{
Sanwei Guo • Georg Müller • Kurt Lehmann • \\ Scherwin Talimi • Gernot Bonkat • Heike Püschel • \\ Thomas Gasser • Alexander Bachmann • Malte Rieken
}

Received: 2 September 2014 / Accepted: 9 February 2015 /Published online: 20 February 2015

(C) Springer-Verlag London 2015

\begin{abstract}
This study aims to compare long-term results of photoselective vaporization of the prostate (PVP) with an $80-\mathrm{W}$ potassium titanyl phosphate (KTP) laser and monopolar transurethral resection of the prostate (TURP) in terms of efficacy, durability, and safety in an adjusted patient population. This prospective, non-randomized bi-center study included 120 (PVP) and 68 (TURP) patients in each arm. Patients were evaluated at 60 months of follow-up. Data from 30 (PVP) and 31 (TURP) patients were available for analysis. The primary outcome measurement was the International Prostate Symptom Score (IPSS) at 5 years. Secondary outcome measurements included voiding symptoms (quality of life (QoL) score), micturition parameters (maximal flow rate, $Q_{\max }$ ), post-void residual (PVR) volume, prostate-specific antigen (PSA) value, and reoperation rate. At study inclusion, voiding symptoms and micturition parameters were comparable between both groups. Age, prostate volume, and the proportion of patients with platelet aggregation inhibition or oral
\end{abstract}

S. Guo $\cdot$ G. Müller $\cdot$ G. Bonkat $\cdot$ H. Püschel $\cdot$ T. Gasser $\cdot$

A. Bachmann $\cdot$ M. Rieken $(\bowtie)$

Department of Urology, University Hospital Basel, Basel,

Spitalstrasse 21, 4031 Basel, Switzerland

e-mail: malte.rieken@usb.ch

K. Lehmann $\cdot$ S. Talimi

Department of Urology, Hospital Baden, Im Ergel 1,

5404 Baden, Switzerland

S. Guo

Department of Urology, Shanghai First People's Hospital affiliated to

Shanghai Jiaotong University, Haining Road 100,

200085 Shanghai, China anticoagulation were significantly higher in the PVP group. No significant difference could be detected between patients available at 60 months and those lost to follow-up in terms of preoperative characteristics in either group. Sixty months postoperatively, the improvement of IPSS, QoL, $Q_{\max }$, and PVR volume showed no significant difference between both groups. PSA reduction was significantly higher after TURP. The reoperation rate due to urethral stricture (PVP, $13 \%$; TURP, none), bladder neck contracture (PVP, $3 \%$; TURP, none), and persisting or recurrent adenoma (PVP, $18 \%$; TURP, $3 \%$ ) was significantly higher after the 80 -W PVP. Eighty-watt PVP leads to comparable functional outcomes to TURP. However, during a long-term follow-up, significantly more reoperations are necessary after PVP with the $80-\mathrm{W}$ KTP laser, suggesting inferior tissue ablation capacity of the 80-W KTP laser.

Keywords Lower urinary tract symptoms $\cdot$ Benign prostate enlargement - Transurethral resection of the prostate . Photoselective vaporization of the prostate . Outcomes . Complications

\section{Introduction}

Transurethral resection of the prostate (TURP) is regarded as the reference technique for the surgical treatment of lower urinary tract symptoms (LUTSs) due to benign prostate enlargement (BPE). Despite the proven efficacy and durability of the procedure, TURP can be associated with significant 
morbidity [1]. In the last decade, several transurethral techniques were developed to overcome the morbidity of TURP while maintaining its efficacy [2,3]. Photoselective vaporization of the prostate (PVP) with a GreenLight laser is one of the established treatment alternatives to TURP [4]. Since its introduction in 2002, the technique underwent several modifications in power output and fiber design [5].

Of the several randomized trials comparing PVP and TURP, the majority demonstrated a comparable functional outcome of PVP with a 120-W HPS laser and TURP in a maximum of 36 months of follow-up [6-10]. Furthermore, intraoperative safety was equal or superior to TURP [6-10]. A recent randomized clinical trial (RCT) could demonstrate a comparable urodynamic deobstruction between 120-W HPS and TURP in patients with prostates $<60 \mathrm{ml}$ after 2 years [10]. In contrast, another recent RCT failed to demonstrate noninferiority of 120-W PVP over monopolar TURP in symptom improvement at 1 year, whereas the superiority of PVP over monopolar TURP regarding hospital stay could be shown [7]. Despite two short-term RCTs comparing PVP with an 80-W potassium titanyl phosphate (KTP) laser and TURP, long-term results of a clinical comparison between $80-\mathrm{W}$ KTP and TURP are still lacking [11, 12].

The aim of the current study was to compare long-term results of patients treated with the 80-W KTP laser to TURP. We hypothesized that, based on the short- and intermediateterm results of our study, PVP and TURP lead to comparable outcomes with respect to symptom improvement, functional outcome, and reoperation rate.

\section{Materials and methods}

Study population

In December 2003, a non-randomized two-center study was initiated to compare the efficacy and safety of PVP with the 80-W KTP laser and monopolar TURP. In this nonrandomized study, data were collected in a prospective database. Six-month and 2-year results were reported previously $[13,14]$. All PVPs were performed with the $80-W$ KTP laser at the University Hospital Basel, whereas all TURPs were performed at the Hospital Baden. Patients in both hospitals were followed up according to the same protocol. Prior to surgery, all patients underwent a standard evaluation including digital rectal examination, urine analysis, transrectal ultrasonography (TRUS) measurement of the prostate, ultrasound evaluation of the kidneys, and blood sample analysis with determination of prostate-specific antigen (PSA). Preoperatively, patients were asked to complete the International Prostate Symptom Score (IPSS) and quality of life (QoL) questionnaire. In case of suspicious digital rectal examination or elevated PSA level, TRUS-guided prostate biopsies were performed to rule out prostate cancer (PCa). Patients with confirmed $\mathrm{PCa}$ or previous transurethral surgery were excluded from the study. Furthermore, patients with known neurogenic bladder disease or neurological disorders were excluded. The use of anticoagulants or platelet aggregation inhibition was not a criterion for exclusion using the 80-W PVP. Patients with indwelling transurethral or suprapubic catheter at surgery, previous retention, and prostate volume $>100 \mathrm{ml}$ were not included in the present adjusted analyses to account for significant differences in baseline characteristic of the initial cohort. There were no age limitations for study inclusion. Preoperatively, all patients gave informed consent. The study as well as the analysis of data was accredited by the local ethics committee, and data storage was performed in consistence with good clinical practice (GCP) guidelines.

\section{Surgical technique}

The techniques of PVP and monopolar TURP were performed as described previously $[13,15]$.

\section{Follow-up}

Patients were followed up at 1, 3, 6, and 12 months postoperatively and yearly thereafter. At every follow-up visit, a standard evaluation was performed including urine analysis, determination of the PSA value, measurement of maximal flow rate $\left(Q_{\max }\right)$, and post-void residual (PVR) volume according the local practical guidelines. Furthermore, the patients were asked to fill out the IPSS/QoL questionnaire on each visit. Adverse events or reoperations were documented at every follow-up visit.

Statistical analysis

All data presented are given as mean \pm standard deviation (SD). Statistical analysis was performed using the SPSS 19.0 statistical software package (SPSS Inc., Chicago, IL, USA). The two-sided $t$ test was applied for the comparison of the mean of two groups and the ANOVA test for the comparison of three or more groups. The Mann-Whitney $U$ test was used to compare two groups of independent nonparametric numerical data. The chi-square test was applied to compare categorical data (complications). A two-sided $p$ value of $<0.05$ was considered statistically significant.

\section{Results}

Baseline data

In total, 269 (PVP) and 127 (TURP) consecutive patients were included in the initial non-randomized study. For analysis, 
data from 257 (PVP) and 104 (TURP) patients were available, respectively. For the present adjusted analyses, 137 (PVP) and 36 (TURP) patients were excluded because of current or previous retention, prostate volume $>100 \mathrm{ml}$, previous transurethral surgery, or neurological disease. After a 60-month follow-up, data from 30 (PVP) and 31 (TURP) patients were available for analysis. At inclusion, patients in the PVP group were significantly older and had a larger prostate volume than those in the TURP group. Ongoing platelet aggregation inhibition or oral anticoagulation was significantly more frequent in the PVP group (Table 1). No significant differences between the group of patients available for 60-month analysis and those lost to follow-up were noticed within either group.

Intra- and perioperative data

Intra- and perioperative data are summarized in Table 2. The duration of surgery was significantly longer with PVP as compared to TURP (72.7 vs. $48.6 \mathrm{~min}, p<0.001)$. The mean of applied energy in the PVP group was $225.8 \mathrm{~kJ}$. The duration of catheterization ( 1.6 vs. 2.5 days, $p<0.001)$ and the duration of hospitalization (5.1 vs. 6.8 days, $p<0.001)$ were significantly shorter after PVP (Table 2).

\section{Functional outcomes}

At a 60-month follow-up, a significant improvement of voiding parameters and symptoms could be noted in both groups. The reduction of IPSS, QoL score, PVR volume, and increase in $Q_{\max }$ showed no significant difference between both groups. The reduction of the PSA value at 60 months was significantly more pronounced after TURP (Table 3).

Table 1 Baseline characteristics

\begin{tabular}{llll}
\hline Parameter & $80-\mathrm{W}$ PVP & TURP & $p$ value \\
\hline Age & $69.7 \pm 8.9$ & $66.4 \pm 8.4$ & $<0.05$ \\
PSA $(\mu \mathrm{g} / \mathrm{l})$ & $3.5 \pm 3.6$ & $3.2 \pm 3.1$ & $\mathrm{~ns}$ \\
Prostate volume $(\mathrm{ml})$ & $52.3 \pm 19.3$ & $44.2 \pm 19.1$ & $<0.05$ \\
IPSS & $19.4 \pm 6.3$ & $18.4 \pm 6.3$ & $\mathrm{~ns}$ \\
QoL & $3.7 \pm 1.7$ & $3.7 \pm 1.3$ & $\mathrm{~ns}$ \\
$Q_{\max }(\mathrm{ml} / \mathrm{s})$ & $8.3 \pm 6.0$ & $10.0 \pm 5.2$ & $\mathrm{~ns}$ \\
PVR $(\mathrm{ml})$ & $119.5 \pm 83.8$ & $95.6 \pm 98.4$ & $\mathrm{~ns}$ \\
ASS $(\%)$ & 25.00 & 4.40 & $<0.01$ \\
Clopidogrel $(\%)$ & 4.20 & 0 & $\mathrm{~ns}$ \\
\hline
\end{tabular}

All values except the values in percentage as mean \pm standard deviation, two-sided $t$ test

$n s$ no significance
Table 2 Intra- and perioperative data

\begin{tabular}{llll}
\hline Parameter & $80-\mathrm{W}$ PVP & TURP & $p$ value \\
\hline Duration of surgery (min) & $72.7 \pm 22.8$ & $48.6 \pm 13.3$ & $<0.001$ \\
Applied energy (kJ) & $225.8 \pm 96.2$ & - & - \\
Duration of catheterization (days) & $1.6 \pm 2.0$ & $2.5 \pm 1.1$ & $<0.001$ \\
Duration of hospitalization (days) & $5.1 \pm 1.8$ & $6.8 \pm 1.5$ & $<0.001$ \\
\hline
\end{tabular}

All values as mean \pm standard deviation, two-sided $t$ test

\section{Reoperations}

Data on the reoperation rate are shown in Table 4. During follow-up, ten of 30 patients $(33.3 \%)$ with available 60 month data after the $80-\mathrm{W}$ PVP had to undergo reoperation compared to one patient (3.2\%) following TURP. Urethral strictures diagnosed in four PVP patients were located in the bulbar urethra and occurred within 1 year after surgery. There was no significant association between the applied energy, duration of surgery, prostate volume, and incidence of urethral strictures. Five patients (17\%) underwent reoperation due to recurrent or persisting adenoma within a follow-up of 5 years. The mean prostate volume of those patients was not significantly higher than that of patients who did not have to undergo repeat surgery. No association between the applied energy, duration of surgery, and reoperation due to adenoma could be detected.

\section{Discussion}

PVP aims to challenge TURP in terms of safety and efficacy. In two previous analyses of our cohort after 6 months and 2 years, the high intra- and perioperative safety of PVP in comparison to TURP could be shown $[13,14]$. The present update of our study is - to our knowledge - the first prospective study comparing PVP and TURP with a long-term follow-up of 5 years. Although this is a non-randomized study, several aspects are worth discussing.

Table 3 Functional outcome with 60 months of follow-up and change of parameters compared to preoperative values

\begin{tabular}{llll}
\hline Parameter & $80-\mathrm{W}$ PVP & TURP & $p$ value \\
\hline PSA $(\mu \mathrm{g} / \mathrm{l})$ & $-1.3 \pm 1.9$ & $-1.9 \pm 1.7$ & 0.001 \\
IPSS & $-12.8 \pm 6.6$ & $-13.5 \pm 6.7$ & $\mathrm{~ns}$ \\
QoL & $-2.4 \pm 1.8$ & $-2.8 \pm 1.5$ & $\mathrm{~ns}$ \\
$Q_{\max }(\mathrm{ml} / \mathrm{s})$ & $6.8 \pm 4.2$ & $12.6 \pm 12.3$ & $\mathrm{~ns}$ \\
PVR $(\mathrm{ml})$ & $-85 \pm 80$ & $-64 \pm 64$ & $\mathrm{~ns}$ \\
\hline
\end{tabular}

All values as mean \pm standard deviation, Mann-Whitney $U$ test $n s$ no significance 
Table 4 Reoperations

\begin{tabular}{|c|c|c|c|c|c|c|}
\hline \multirow[t]{2}{*}{ Parameter } & \multicolumn{3}{|c|}{ All patients (80-W PVP, 120; TURP, 68) } & \multicolumn{3}{|c|}{ Patients with completed 60-month follow-up (80-W PVP, 30; TURP, 31) } \\
\hline & 80-W PVP (\%) & TURP $(\%)$ & $p$ value & 80-W PVP $(\%)$ & TURP $(\%)$ & $p$ value \\
\hline Urethral stricture & $7(6)$ & $1(2)$ & ns & $4(13)$ & 0 & - \\
\hline Bladder neck contracture & $3(3)$ & 0 & - & $1(3)$ & 0 & - \\
\hline Persisting/recurrent adenoma & $10(8)$ & $2(3)$ & $<0.05$ & $5(17)$ & $1(3)$ & ns \\
\hline All reoperations & $20(17)$ & $3(4)$ & 0.001 & $10(33)$ & $1(3)$ & $<0.05$ \\
\hline
\end{tabular}

All values in numbers (percentages), chi-square test

$n s$ no significance

In our study, we found that both techniques lead to a comparable improvement of voiding parameters and symptoms. This observation is in par with other prospective trials comparing PVP and TURP [6-11]. While the functional outcome during follow-up was comparable, PSA reduction was more pronounced after TURP $[6,7]$. This suggests a higher tissue ablation after TURP in comparison to PVP with the $80-\mathrm{W}$ laser. The reduced PSA reduction after the 80 -W PVP is consistent with our data, where the reduction of PSA at 5 years was significantly higher after TURP.

In our study, $33 \%$ (80-W PVP) and $3 \%$ (TURP) of the patients had to undergo reoperation during follow-up. The reoperation rate was due to a higher rate of recurrent or persisting adenoma as well as urethral strictures. All patients who developed a urethral stricture during follow-up were operated with a $26 \mathrm{~F}$ cystoscope, which was replaced by a $22.5 \mathrm{~F}$ cystoscope with a separate channel for the laser fiber at our center in April 2006. In a previously published analysis of 500 patients who underwent PVP with the 80-W KTP laser at our center, a significant association between cystoscope size and incidence of urethral strictures was described ( $1 \%$ with $22.5 \mathrm{~F}$ vs. $6 \%$ with $26 \mathrm{~F}, p<0.001$ ) [16]. This association is supported by the observation that none of the patients in the current cohort operated with the $22.5 \mathrm{~F}$ instrument did experience a urethral stricture.

In our analysis, five of $30(17 \%)$ patients with available data at 60 months had to undergo reoperation due to persisting or recurrent adenoma. In RCTs available with the 80 -W laser, the reoperation rate after 6 and 12 months was reported to be 23.1 and $16.9 \%$, respectively [11, 12]. Al-Ansari et al. report reoperation rates of $18.5 \%$ (PVP) and $5.5 \%$ (TURP) during a 3-year follow-up with the 120-W HPS laser, whereas Capitán et al. report reoperation rates of $2 \%$ (PVP) and $12 \%$ (TURP) within 2 years after surgery. Notably, no redo PVP/TURP was necessary in the cohort of Capitán et al., while no urethral stricture was reported by Al-Ansari et al. In our study, three of five patients had to be reoperated within the first 6 months after surgery. Thus, an insufficient deobstruction during PVP may be postulated. This could, in part, be explained by the learning curve, as two of the three operations were performed by novices. Furthermore, the higher reoperation rate due to recurrent or persisting adenoma suggests a lower tissue ablation capacity of the 80-W KTP laser in comparison to TURP. This is supported by the fact that PSA reduction after TURP is significantly higher than after PVP with the $80-\mathrm{W}$ laser. In a previous analysis of our cohort, we could show that the PSA reduction is significantly higher with the current $180-\mathrm{W}$ system compared to the former systems [5]. Nevertheless, it is important to notice that around $40 \%$ of our $80-\mathrm{W}$ patients were under treatment with platelet aggregation inhibition or oral anticoagulation so that at least a part of these patients might only be safely operated with PVP. In addition, no significant association between the incidence of reoperation and the platelet aggregation inhibition/oral anticoagulation was detected. Thus, even in consideration of the higher reoperation rate, PVP may be regarded as a valuable treatment option for patients with higher cardiovascular risks [17].

The duration of hospitalization in our study is relatively long compared to other studies in this field [6,7]. This is primarily related to aspects of the health-care system in Switzerland. At the time of study performance, hospitals were reimbursed on a per day basis and not per case basis. Thus, the admission time is generally longer compared to what is usually reported from such patient cohorts $[6,7]$.

We are aware of the limitations or our study. First, the high rate of patients who were lost to follow-up at 60 months or did refuse further participation is notably high and seen in a multitude of non-randomized studies. As this is a bi-center study with own local policy in postoperative management, underreporting of events is a potential bias. However, the statistical analysis of patients with available follow-up and those lost to follow-up did not reveal any significant differences between the groups. Thus, the remaining patients and their postoperative course may be regarded as representative. Another limitation is the lack of randomization. Since this is a two-center study with experience in either performed surgical technique, a high grade of expertise can be postulated. In addition, the current series covers patients treated with the 80-W KTP laser, which has been replaced by the higherpowered 120 - or $180-\mathrm{W}$ systems in the majority of the centers. 
Nevertheless, 5-year outcomes of PVP are limited, and thus, our data provide relevant data. Moreover, mostly the patients with smaller prostates in the TURP group and mostly the patients with larger prostates and more comorbidities in the 80-W PVP group were treated. This is an important difference making the analyses by a direct comparison of the groups prone to bias. Nevertheless, as these differences reflect the real-life setting in urological practice, our results offer an important source of information.

\section{Conclusions}

The 80-W PVP and TURP show a comparable long-term improvement of voiding symptoms and micturition parameters. The duration of catheterization and hospitalization is significantly shorter after the $80-\mathrm{W}$ PVP. The reoperation rate due to a persisting adenoma is significantly higher after PVP. This suggests an inefficient and insufficient tissue ablation capacity of the former 80 -W KTP laser.

Conflict of interest Alexander Bachmann is a consultant for American Medical Systems (AMS) and a principal investigator of a study comparing photoselective vaporization of the prostate with the GreenLight XPS $^{\mathrm{TM}}$ laser system and transurethral resection of the prostate for the treatment of benign prostatic hyperplasia (The Goliath Study). Heike Püschel is a study nurse and partly paid by AMS. The remaining authors declare that they have no conflict of interest.

\section{References}

1. Reich O, Gratzke C, Bachmann A, Seitz M, Schlenker B, Hermanek P, Lack N, Stief CG, Urology Section of the Bavarian Working Group for Quality A (2008) Morbidity, mortality and early outcome of transurethral resection of the prostate: a prospective multicenter evaluation of 10,654 patients. J Urol 180(1):246-249. doi:10.1016/j.juro.2008. 03.058

2. Ahyai SA, Gilling P, Kaplan SA, Kuntz RM, Madersbacher S, Montorsi F, Speakman MJ, Stief CG (2010) Meta-analysis of functional outcomes and complications following transurethral procedures for lower urinary tract symptoms resulting from benign prostatic enlargement. Eur Urol 58(3):384-397. doi:10.1016/j.eururo. 2010.06.005

3. Rieken M, Bachmann A (2014) Laser treatment of benign prostate enlargement - which laser for which prostate? Nat Rev Urol 11(3): 142-152. doi:10.1038/nrurol.2014.23

4. Oelke M, Bachmann A, Descazeaud A, Emberton M, Gravas S, Michel MC, N'Dow J, Nordling J, de la Rosette JJ, European Association of U (2013) EAU guidelines on the treatment and follow-up of non-neurogenic male lower urinary tract symptoms including benign prostatic obstruction. Eur Urol 64(1):118-140. doi: 10.1016/j.eururo.2013.03.004

5. Rieken M, Bonkat G, Muller G, Wyler S, Mundorff NE, Puschel H, Gasser T, Bachmann A (2013) The effect of increased maximum power output on perioperative and early postoperative outcome in photoselective vaporization of the prostate. Lasers Surg Med 45(1): 28-33. doi:10.1002/1sm.22108
6. Al-Ansari A, Younes N, Sampige VP, Al-Rumaihi K, Ghafouri A, Gul T, Shokeir AA (2010) GreenLight HPS 120-W laser vaporization versus transurethral resection of the prostate for treatment of benign prostatic hyperplasia: a randomized clinical trial with midterm follow-up. Eur Urol 58(3):349-355. doi:10.1016/j.eururo.2010.05.026

7. Capitan C, Blazquez C, Martin MD, Hernandez V, de la Pena E, Llorente C (2011) GreenLight HPS 120-W laser vaporization versus transurethral resection of the prostate for the treatment of lower urinary tract symptoms due to benign prostatic hyperplasia: a randomized clinical trial with 2-year follow-up. Eur Urol 60(4):734-739. doi:10.1016/j.eururo.2011.05.043

8. Kumar A, Vasudeva P, Kumar N, Nanda B, Jha SK, Mohanty NK (2013) A prospective randomized comparative study of monopolar transurethral resection of prostate (TURP), bipolar TURP and photoselective vaporization of prostate in patients presenting with benign prostatic obstruction - a single center experience. J Endourology/Endourological Soc. doi:10.1089/end.2013.0216

9. Lukacs B, Loeffler J, Bruyere F, Blanchet P, Gelet A, Coloby P, De la Taille A, Lemaire P, Baron JC, Cornu JN, Aout M, Rousseau H, Vicaut E, Group RS (2012) Photoselective vaporization of the prostate with GreenLight 120-W laser compared with monopolar transurethral resection of the prostate: a multicenter randomized controlled trial. Eur Urol 61(6):1165-1173. doi:10.1016/j.eururo.2012.01.052

10. Pereira-Correia JA, de Moraes Sousa KD, Santos JB, de Morais Perpetuo D, Lopes-da-Silva LF, Krambeck RL, Muller VJ, Vaz FP (2012) GreenLight HPS 120-W laser vaporization vs transurethral resection of the prostate $(<60 \mathrm{~mL})$ : a 2 -year randomized doubleblind prospective urodynamic investigation. BJU Int 110(8):11841189. doi:10.1111/j.1464-410X.2011.10878.x

11. Bouchier-Hayes DM, Van Appledorn S, Bugeja P, Crowe H, Challacombe B, Costello AJ (2010) A randomized trial of photoselective vaporization of the prostate using the 80 -W potassium-titanyl-phosphate laser vs transurethral prostatectomy, with a 1-year follow-up. BJU Int 105(7):964-969. doi:10.1111/j. 1464-410X.2009.08961.X

12. Horasanli K, Silay MS, Altay B, Tanriverdi O, Sarica K, Miroglu C (2008) Photoselective potassium titanyl phosphate (KTP) laser vaporization versus transurethral resection of the prostate for prostates larger than $70 \mathrm{~mL}$ : a short-term prospective randomized trial. Urology 71(2):247-251. doi:10.1016/j.urology.2007.09.017

13. Bachmann A, Schurch L, Ruszat R, Wyler SF, Seifert HH, Muller A, Lehmann K, Sulser T (2005) Photoselective vaporization (PVP) versus transurethral resection of the prostate (TURP): a prospective bicentre study of perioperative morbidity and early functional outcome. Eur Urol 48(6):965-971. doi:10.1016/j.eururo.2005.07.001, discussion 972

14. Ruszat R, Wyler SF, Seitz M, Lehmann K, Abe C, Bonkat G, Reich O, Gasser TC, Bachmann A (2008) Comparison of potassiumtitanyl-phosphate laser vaporization of the prostate and transurethral resection of the prostate: update of a prospective non-randomized two-centre study. BJU Int 102(10):1432-1438. doi:10.1111/j.1464410X.2008.07905.x, discussion 1438-1439

15. Gomez Sancha F, Bachmann A, Choi BB, Tabatabaei S, Muir GH (2007) Photoselective vaporization of the prostate (GreenLight PV): lessons learnt after 3500 procedures. Prostate Cancer Prostatic Dis 10(4):316-322. doi:10.1038/sj.pcan.4500989

16. Ruszat R, Seitz M, Wyler SF, Abe C, Rieken M, Reich O, Gasser TC, Bachmann A (2008) GreenLight laser vaporization of the prostate: single-center experience and long-term results after 500 procedures. Eur Urol 54(4):893-901. doi:10.1016/j.eururo.2008.04.053

17. Ruszat R, Wyler S, Forster T, Reich O, StiefCG, Gasser TC, Sulser T, Bachmann A (2007) Safety and effectiveness of photoselective vaporization of the prostate (PVP) in patients on ongoing oral anticoagulation. Eur Urol 51(4):1031-1038. doi:10.1016/j.eururo. 2006.08.006, discussion 1038-1041 